\title{
Improving Adherence: A Systematic Review of Text Messaging in HIV Treatment
}

\author{
Whitney B. Sparks* and Alice L. March \\ Capstone College of Nursing, University of Alabama, USA
}

\begin{abstract}
Key point 1: The obstacles that stand in the way of the Healthy People 2020 objective HIV-22, to increase viral suppression among HIV positive people, could be overcome by utilization of text messaging to encourage treatment adherence.

Key point 2: This systematic review of recent studies identified receptiveness, positive behavioral modifications, and improved treatment measures as important variables related to text messaging for appointment and treatment reminders.
\end{abstract}

Key point 3: Text messaging used in high-risk HIV positive populations to send daily medication reminders, appointment reminders, and viral load testing reminders yields improved ART adherence and is key in meeting treatment goals.

\section{Introduction}

There are over one million people in the United States (US) who are living with HIV and $15 \%$ are not aware that they have the virus [1]. Within the US, the yearly incidence remained stable from 2012 to 2016. However, in 2017 that incidence increased to38, 739 new cases [2]. Of those cases, there is a disproportionality percent of men who have sex with men (MSM) at 66\% [2].

Most new HIV cases exist in populations ranging from 13 to 34-years-old, and adolescents have a higher risk of being unaware of their HIV status [3]. People living with HIV are commonly from high-risk populations and are often more difficult to retain for routine care and preventative treatment [4]. The CDC identifies these high-risk groups as African Americans, injection drug users, Latin Americans, gay and bisexual men of all races and ethnicities, and transgendered people [5].

The HIV prevalence in urban and low-income areas in America exceeds the cutoff defining definition of an HIV epidemic [3]. In addition, there are increased rates of HIV in poverty-ridden areas. High-risk populations face barriers to adherence to antiretroviral therapy (ART) which inhibits effective HIV care because viral loads (VL) are not adequately suppressed. Barriers to initial and ongoing treatment include but are not limited to depression and other mental illnesses, unreliable transportation to office visits, cost, insufficient health insurance, social support, and forgetting to take medications [4].

Healthy People 2020 have multiple objectives that pertain to HIV management. The goal of objective HIV-22 is to increase viral suppression among HIV positive people who are 13-years of age or older [6]. The obstacles that stand in the way of this goal could be overcome by utilization of cellular technology, such as text messaging, to encourage treatment adherence [7].

Adherence to ART is essential for every person living with HIV as it is the only means for effective viral load suppression. Yet only $51 \%$ of people living with HIV/AIDS (PLWHA) in the US have met the goal of VL suppression [1]. When the $\mathrm{VL}$ is adequately suppressed progression to AIDS is slowed, a higher quality of life is reported, and the risk of transmission during sex is lower [8].

To increase ART adherence and decrease viral load text messaging could be used in high-risk populations to send daily medication reminders, appointment reminders, and viral load (VL) testing reminders. The U.S. Department of Health and Human Services, Health Resources and Services Administration identified text messaging as a necessary method of intervention to encourage ART and HIV treatment adherence [9]. This method can be particularly helpful in HIV-positive

*Corresponding author: Whitney B. Sparks, Capstone College of Nursing, University of Alabama, P.O. Box 676 Belmont, MS 38827, USA

Accepted: March 01, 2021

Published online: March 03, 2021

Citation: Sparks WB, March AL (2021) Improving Adherence: A Systematic Review of Text Messaging in HIV Treatment. Clin J HIV AIDS 5(1):46-50 
youth and young adults due to increased rates of cell phone use and proficiency [7].

\section{Methods}

The common problem identified for this review was lack a of ART and HIV treatment adherence. The intervention pertinent to this review is utilization of text messaging to encourage treatment adherence. A comparison to this intervention would be the absence of the use of text messaging for treatment reminders and simply relying on the traditional standard of care (SOC). The current SOC involves only treatment and medication education during office visits with the patient.

A review of the literature concerning HIV therapy, ART adherence, and cell phone technology revealed important studies concerning the benefits of text messaging to support treatment adherence among HIV-positive people. The review followed the Preferred Reporting Items for Systematic Reviews and Meta-Analysis (PRISMA) guidelines [10].

The search strategies included only empirical data-based studies published in the last five years and were identified using NCBI/PubMed, MEDLINE, Web of Science, and CINAHL databases (Figure 1). The MeSH terms used included combinations of HIV treatment adherence, antiretroviral therapy, cell phone, eHealth, text messaging, and short messaging service (SMS). The four databases yielded a total of 2,184 studies. After excluding duplicates this number was decreased to 1,698 . The remaining studies were limited to full text articles published in the last five years which resulted in 675 articles. Articles were then screened for irrelevant titles and studies that did not include smart phone technologies. Once this filter was applied 23 articles remained. Of those articles nine were excluded due to being qualitative studies, and two systematic reviews were excluded because they included data obtained from qualitative designs. This left 12 studies included in this systematic review which synthesizes issues of receptiveness, positive behavioral modifications, and a variety of improved treatment measures detailed in this manuscript (see Figure 1).

\section{Significant Findings of Reviewed Studies}

Using the population, intervention, comparison, outcome, and study design (PICOS) framework the population evaluat-

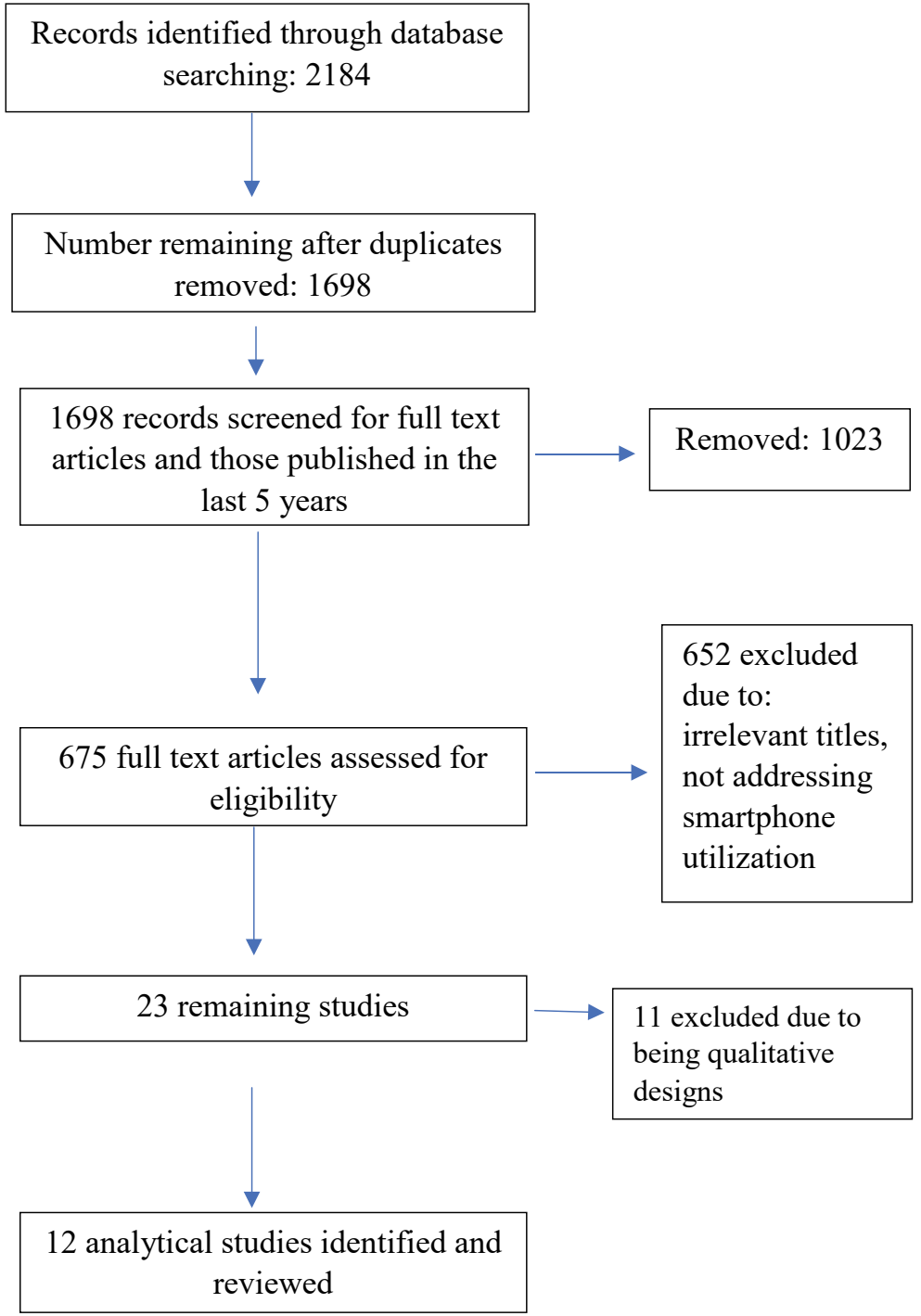

Figure 1: Flow of information through phases of review. 
ed was HIV-positive patients. To assess the current state of the literature related to PLWHA this guided review included ten randomized controlled trials (RCT) with evaluations of PLWHA. Participants resided in Australia [11], China [12], the US [13-16], India [17], and Africa [18-20]. Additionally, the review included a meta-analysis [21] and a systematic review [22] which included an analytic data review of HIV positive populations residing in Asia Pacific, Europe, the US, and Africa.

Reported stigma in relation to personal attributes was the only identified independent factor associated with statistically significant consistent adherence to ART [11]. After six weeks of text message ART reminders to MSM in India ART adherence improved from $43.5 \%$ to $82 \%$ [11]. Similarly, Mayer and Fontelo [21] found that text message reminders considerably improved ART adherence. Statistically significant improvements in physiologic measures and decreased rates of non-attendance were associated with text message reminders [21]. The only RCT that found that text messaging services did not significantly improve retention in HIV treatment included PLWHA in Kenya. However, it is clinically relevant to note that $79 \%$ of the intervention group was retained in care compared to $81 \%$ in the control group [20].

Retention in care was not the only positive behavioral modification found with text messaging. At the end of a sixmonth study the intervention group who received reminders had fewer reports of anal sex without condoms during the past month compared to the control group [17].

Text messaging resulted insignificant improvements in ART adherence among youth living with HIV [14]. When participants with a greater than $90 \%$ adherence rate were compared by group, the text messaging group demonstrated greater increases in adherence rates at 3 and 6 months. Those increases included $64 \%$ and $61 \%$ at 3 and 6 months (respectively) in the intervention group, and $43 \%$ and $51 \%$ at 3 and 6 months in the control group (respectively).

The Wise pill plus text messaging intervention was beneficial in ART adherence with a Ugandan group [19]. Adherence findings were $11.1 \%$ higher in the intervention group when compared to the control group, and medication lapses lasting 48 hours or greater were less frequent in the intervention group. Likewise, a nine-month RCT supported the combination of Wise pill and text messaging among PLWHA in Nanning, China [12]. The percent of participants maintaining optimal adherence (defined as $\geq 95 \%$ ) pre-trial was 63.5 in the intervention group and 58.9 in the control group. Post intervention, the percent in the intervention group increased to 87.3, while the control group percent declined to 51.8. Post intervention, optimal adherence increased by $23.8 \%$ in the intervention group and declined $7.1 \%$ in the control group, and the mean adherence was $96.2 \%$ in the intervention group compared to $89.1 \%$ in the control group [12].

Another RCT measured three objectives from an intervention utilizing daily ART text messaging [15]. The definition of ART adherence was a calculated score measured by pharmacy fill rate, proportion of missed visits, and rate of reported substance abuse. There was an increase in overall ART adher- ence from $66 \%$ to $85 \%$ in the intervention group compared to as smaller increase from $62 \%$ to $71 \%$ in the control group. Missed visit rates in the intervention group decreased from $23 \%$ to $9 \%$ compared to a smaller decrease of $31 \%$ to $28 \%$ in the control group.

The final outcomes of three longitudinal research are still pending; however, these are hypothesized to be well received, positively influence behavioral modifications for PLWHA, and further support text messaging as an effective tool to promote HIV treatment adherence $[13,16,18]$.

\section{Discussion}

This research identified receptiveness, positive behavioral modifications, and a variety of improved treatment measures attained with the utilization of text messaging. In addition, the variety of studies from around the world demonstrates a possible universal approach to increasing viral suppression through increased adherence.

\section{Receptiveness}

Several RCTs support findings that PLWHA are receptive to the use of text messaging during HIV treatment $[11,16,17]$. In the RCT conducted in India, HIV-positive MSM and male sex workers were receptive to text messaging. This finding was supported by a $98 \%$ retention rate and a $96 \%$ consistent involvement rate over the six-month study [17]. Similarly, MSM in Australia are also receptive to text messaging from community support networks, which introduces the idea that this type of intervention can exist outside of the provider-patient relationship [11]. Likewise, text messaging was well received by U.S. youth living with HIV who were age 16 to 29 [14]. At the end of that 6-month RCT involving text message ART reminders $95 \%$ of participants reported satisfaction with text messaging and $100 \%$ agreed that they would recommend it to a friend.

\section{Behavioral modifications}

Results from three studies found that text messaging positively influences behavioral modifications in high-risk HIV populations $[17,21,22]$. At the end of a six-month study the text messaging group reported fewer instances of anal sex without condoms during the past month [17]. Contrasting those data, Mayer and Fontelo did not find that text messaging reduced the rate of high-risk sexual acts; however, they did find improved retention rates. Increases in care retention and reported condom use were also identified as conclusions in the systematic review [22].

Care retention rates are also a concern for effective HIV treatment. WelTel Retain, a text messaging system, was used in a study among HIV positive people in Kenya [20]. That trial did not demonstrate improved retention in HIV care. However, retention rates in HIV treatment as a behavioral standard has pending research data. An ongoing longitudinal study in progress in urban and rural Kenya using WelTel services to evaluate improvement in the prevention of mother to child transmission (PMTCT) by utilizing weekly 2-way text messaging to retain mothers and infants in HIV care until the child is 24-months-old [18]. The hypothesis is that this will signifi- 
cantly reduce pediatric HIV infections. Likewise, in a 12-month prospective study in progress in the US researchers hypothesize that text messaging will improve retention because the formatting of the message will be focused on psychosocial outcomes [13].

\section{Improved treatment measures}

The meta-analysis and systematic review evaluated in this literature review provided insight into a variety of study designs. The systematic review [22] identified 16 studies and the meta-analysis [21] identified 34 articles through database searches. Study designs included RCTs, cross-sectional, and cohort inquiries. The 34 articles identified in the meta-analysis evaluated 13,176 participants. The overall conclusion was that text messaging considerably improved ART adherence and physiologic measures such as viral load [21].

The 16 studies identified in the systematic review [22] included publications from 2010 to 2016 concerning eHealth and HIV treatment in the Asia-Pacific region and evaluated a total of 8,869 people. In that review, eHealth involved text messaging and web-based applications accessible by phones with internet access. The review presented two categories; seven studies focused retention in care, and nine focused on treatment and adherence. Seven studies were cross-sectional, seven were RCTs, and the remaining two were cohort studies. Of the 16 studies, five RCTs had a strong global rating or methodological quality due to the strength of study designs, data collection, attention to confounders, and study blinding. Thirteen of those studies demonstrated improved outcomes in a variety of HIV treatment areas including self-reported decreased missed doses, 95\% or greater ART adherence, increase in retesting, and a decrease in viral loads. In contrast, the review also identified two studies that revealed no significant improvement in increased HIV testing, and one that identified no improvement in ART adherence even with bi-weekly contact [22].

An RCT conducted in Australia enrolled a small sample group of PLWHA ( $n=98)$. The sample included predominantly MSM (85.5\%) [11]. Participants received text messaging and answered questions concerning independent factors associated with non-adherence to ART. Reported stigma concerning personal attributes was the only statistically significant independent factor. At the beginning of the six-week study 43.5\% had interrupted ART treatment; however, by the last week of the study, the text messaging group improved to an adherence rate of $82 \%$ [11].

The Wise pill medication monitoring device used with text messaging was beneficial in improving ART adherence $[12,19]$. A group of PLWHA in China, who were considered high risk for non-adherence, were evaluated for 9 months using the monitoring device coupled with text messaging which encouraged adherence and pill reminders when medications were missed [12]. Likewise, PLWHA in Uganda who were newly initiating ART were included in a 3-month study using both Wise pill and adherence encouraging text messaging [19]. The intervention groups in both studies demonstrated significant improvement in ART adherence $[12,19]$.
Similarly, in an ongoing 12-month two-arm controlled efficacy trial, researchers hypothesize that daily text message reminders, along with the use of Wise pill, will improve ART adherence among HIV-positive youth [16]. This hypothesis may be based on a completed study in which participants using Wise pill had significant decreases in viral loads [12]. The ongoing study by Mimiaga, et al. includes nearly 200 American PLWHA who are ages 16-29 and reside in two large cities in New England and the Mid-West. These participants were recruited via community events, social media, and college/ university outreach centers. Inclusion criteria were that the person had been prescribed ART and was having self-reported administration difficulties. Outcome measures include assessment of daily device access, self-reported adherence, and viral load measurements [16].

Youth living with HIV, MSM, and people who abuse substances are among those considered as high-risk HIV populations and are difficult to retain in care $[5,14]$. A completed RCT of high-risk youth living with HIV in the U.S. mid-west found that 2-way messaging was beneficial in ART adherence [14]. Similarly, among nonurban HIV positive people who abused substances and who were not adherent to ART participant-designed daily text messaging improved ART adherence by $19 \%$ [15]. Ongoing research to support text messaging is pending [14]. In that study, among high-risk and difficult to retain PLWHA who reside in San Francisco researchers hypothesize that viral load will decrease with thrice weekly text messaging [14].

\section{Gaps and limitations}

Although a variety of studies were identified there are gaps and limitations noted within those articles. Multiple study samples were comprised of a larger percent of MSM and therefore may not have been representative all HIV-positive populations $[11,14-16,19]$. Eleven of the sixteen articles identified in the systematic review had quality limitations due to study designs, data collection, and attrition rates [22]. Similarly, the RCTs identified by the current systematic review had comparable limitations. Small sample sizes $[11,19]$ as well as samples limited to metropolitan areas $[12,13,17]$ existed and this may create gaps in accuracy when using text messaging among other PLWHA. Most research that identified using smart phone technology examined MSM; therefore, more studies utilizing text messaging for other populations of PLWHA would be beneficial. Excluding the studies that used Wise pill, investigations explicitly relied on self-reported measures of adherence; thus, introducing the possibility of bias.

\section{Conclusion}

Text messaging used in high-risk HIV positive populations to send daily medication reminders, appointment reminders, and viral load testing reminders has yielded improved ART adherence and has been key in meeting treatment goals. This review of RCTs, a meta-analysis, and one systematic review supports the hypothesis that text messaging is beneficial in HIV management. Care interventions using text messaging were well received among MSM and other populations of PLWHA. Furthermore, those interventions promoted positive 
behavioral modifications that may help prevent the spread of HIV by decreasing viral load $[11,17]$.

\section{Funding}

This research did not receive any specific grant from funding agencies in the public, commercial, or not-for-profit sectors.

\section{Declaration of Interests}

None.

\section{References}

1. https://www.hiv.gov/hiv-basics/overview/data-and-trends/statistics

2. https://www.cdc.gov/hiv/statistics/overview/ataglance.html

3. Denning P, DiNenno E (2017) Communities in crisis: Is there a generalized HIV epidemic in impoverished urban areas of the United States?

4. Yehia BR, Stewart L, Momplaisir F, et al. (2015) Barriers and facilitators to patient retention in HIV care. BMC Infect Dis 15: 246.

5. Centers for Disease Control and Prevention (2015) Populations at greatest risk.

6. Office of Disease Prevention and Health Promotion (2019) HIV22 increase the proportion of persons aged 13 years and older with diagnosed HIV who are virally suppressed.

7. Venables E, Ndlovu Z, Munyaradzi D, et al. (2019) Patient and health-care worker experiences of an HIV viral load intervention using SMS: A qualitative study. PLoS One 14: e0215236.

8. https://aidsinfo.nih.gov/understanding-hiv-aids/factsheets/21/54/hiv-treatment-adherence

9. https://hab.hrsa.gov/sites/default/files/hab/Publications/factsheets/improvingcareyouthhiv.pdf

10. http://www.prisma-statement.org/

11. Mao L, Buchanan A, Wong HTH, et al. (2018) Beyond mere pill taking: SMS reminders for HIV treatment adherence delivered to mobile phones of clients in a community support network in Australia. Health Soc Care Community 26: 486-494.

12. Sabin LL, Desilva MB, Gill CJ, et al. (2015) Improving adherence to antiretroviral therapy with triggered real-time text message reminders. JAIDS Journal of Acquired Immune Deficiency Syndromes 69: 551-559.
13. Christopoulos KA, Riley ED, Tulsky J, et al. (2014) A text messaging intervention to improve retention in care and virologic suppression in a U.S. urban safety-net HIV clinic: Study protocol for the Connect4Care (C4C) randomized controlled trial. BMC Infect Dis 14: 718.

14. Garofalo R, Kuhns L, Hotton A, et al. (2016) A randomized controlled trial of personalized text message reminders to promote medication adherence among HIV-positive adolescents and young adults. AIDS Behav 20: 1049-1059.

15. Ingersoll KS, Dillingham RA, Hettema JE, et al. (2015) Pilot RCT of bidirectional text messaging for ART adherence among nonurban substance users with HIV. Health Psychol 34: 1305-1315.

16. Mimiaga MJ, Kuhns LM, Biello KB, et al. (2018) Positive STEPS - a randomized controlled efficacy trial of an adaptive intervention for strengthening adherence to antiretroviral HIV treatment among youth: Study protocol. BMC Public Health 18: 867.

17. Mimiaga MJ, Thomas B, Biello K, et al. (2017) A pilot randomized controlled trial of an integrated in-person and mobile phone delivered counseling and text messaging intervention to reduce HIV transmission risk among male sex workers in Chennai, India. AIDS Behav 21: 3172-3181.

18. Awiti P, Grotta A, van der Kop M, et al. (2016) The effect of an interactive weekly mobile phone messaging on retention in prevention of mother to child transmission (PMTCT) of HIV program: Study protocol for a randomized controlled trial (WELTEL PMTCT). BMC Med Inform Decis Mak 16: 86.

19. Haberer JE, Musiimenta A, Atukunda EC, et al. (2016) Short message service (SMS) reminders and real-time adherence monitoring improve antiretroviral therapy adherence in rural Uganda. AIDS 30: 1295-1300.

20. van der Kop ML, Muhula S, Nagide PI, et al. (2018) Effect of an interactive text-messaging service on patient retention during the first year of HIV care in Kenya (WelTel Retain): An open-label, randomized parallel-group study. Lancet Public Health 3: e143-e152.

21. Mayer JE, Fontelo P (2017) Meta-analysis on the effect of text message reminders for HIV-related compliance. AIDS Care 29: 409-417.

22. Purnomo J, Coote K, Mao L, et al. (2018) Using eHealth to engage and retain priority populations in the HIV treatment and care cascade in the Asia-Pacific region: A systematic review of literature. BMC Infect Dis 18: 82.

DOI: $10.36959 / 695 / 569$

Copyright: (C) 2021 Sparks et al. This is an open-access article distributed under the terms of the Creative Commons Attribution License, which permits unrestricted use, distribution, and reproduction in any medium, provided the original author and source are credited. 